\title{
Promoter Hypermethylation of Tumor Suppressor Genes Located on Short Arm of the Chromosome 3 as Potential Biomarker for the Diagnosis of Nasopharyngeal Carcinoma
}

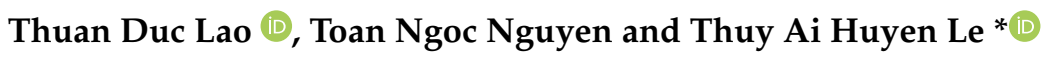 \\ Department of Pharmaceutical and Medical Biotechnology, Faculty of Biotechnology, Ho Chi Minh City Open \\ University, Ho Chi Minh City 700000, Vietnam; thuan.ld@ou.edu.vn (T.D.L.); nngoctoan2000@gmail.com (T.N.N.) \\ * Correspondence: thuy.lha@ou.edu.vn
}

check for updates

Citation: Lao, T.D.; Nguyen, T.N.; Le, T.A.H. Promoter Hypermethylation of Tumor Suppressor Genes Located on Short Arm of the Chromosome 3 as Potential Biomarker for the Diagnosis of Nasopharyngeal Carcinoma. Diagnostics 2021, 11, 1404 https://doi.org/10.3390/diagnostics 11081404

Academic Editor: Paweł Golusiński

Received: 21 June 2021

Accepted: 2 August 2021

Published: 3 August 2021

Publisher's Note: MDPI stays neutral with regard to jurisdictional claims in published maps and institutional affiliations.

Copyright: (c) 2021 by the authors. Licensee MDPI, Basel, Switzerland. This article is an open access article distributed under the terms and conditions of the Creative Commons Attribution (CC BY) license (https:/ / creativecommons.org/licenses/by/ $4.0 /)$.

\begin{abstract}
DNA methylation, the most common epigenetic alteration, has been proven to play important roles in nasopharyngeal carcinoma (NPC). Numerous tumor suppressor genes located on the chromosome 3p, particularly in the region of 3p21, are frequently methylated in NPC, thus suggesting great potential for diagnosis of NPC. In this review, we summarize recent findings of tumor suppressor genes on chromosome 3 that likely drive nasopharyngeal tumor development and progression, based on previous studies related to the hypermethylation of these target genes. Better understanding will allow us to design further experiments to establish a potential test for diagnosis of NPC, as well as bring about methylated therapies to improve the treatment of NPC.
\end{abstract}

Keywords: nasopharyngeal carcinoma; chromosome 3p; methylation; diagnosis

\section{Introduction}

Cancer is a serious global health problem and the second leading cause of death globally, with an estimated 9.6 million deaths in 2018. It was noted that the number of new cases will reach 23.6 million by 2030 [1]. Nasopharyngeal carcinoma (NPC) is the most common cancer in the area of head and neck cancer. NPC has unbalanced geographical differences in distribution, which gravitates toward Asia. Asian countries account for $84.62 \%$ of the global burden of NPC. Similarly, the highest mortality rate has also been observed in Asian countries, accounting for $85.36 \%$ of the global mortality of NPC. In recent decades, even though improvements in nasopharyngeal cancer treatment have made great progress, diagnosis at the advanced stage of tumors often leads to treatment failure as well as reduction in the survival of patients. Early diagnosis represents a beneficial opportunity to improve the treatment results, resulting in a high cure rate [2]. The paucity or nonspecific symptoms of the early stage of NPC, as well as the deeply seated location of the nasopharynx, are major obstacles leading to the diagnosis of NPC at the advanced stage. Finding the effective biomarkers for early diagnosis of NPC will contribute significantly to improving survival and will guide the choice of subsequent therapy, particularly in the countries with high incidence.

Providing information about the etiological factors in the prevention program is very important. At present, much effort has been made to identify early biomarkers by focusing on the etiological factors that lead to nasopharyngeal tumorigenesis. NPC presents as a multiple etiological factors disease that is caused by: (1) the infection of Epstein-Barr virus (EBV); (2) environmental factors; and (3) genetic susceptibility [3]. Recent advances in the field of molecular and cytogenetic studies have shown that genetic and epigenetic events play key roles at all stages of tumorigenesis and promote cancer progression [4]. It has been suggested that the mechanism of epigenetic alteration may act as the initiating event for various human cancers, including NPC, through regulating cell signal pathways [4-7]. Epigenetics, originally defined by C.H. Waddington (1942), refers to "the causal interactions 
between genes and their products, which bring the phenotype into being", and involves the following main categories: DNA methylation, covalent histone modifications, and noncoding RNAs $[4,8]$. Unlike genetic mutation, epigenetic alterations can be reversed to their normal state, thereby making such initiatives promising therapeutic biomarkers [4]. DNA methylation, which is the most common epigenetic alteration, is a covalent modification of $5^{\prime}$ cytosine located at a CpG dinucleotide by adding a methyl group in the 5th carbon of the ring using S-adenosyl methionine as a methyl donor $[4,9,10]$. The alterations of DNA hypermethylation in the promoter region or the first exon of the tumor suppressor gene (TSG) lead to its inactivation. Decreasing of TSG is proven to be significantly associated with tumorigenesis. Therefore, understanding the characteristics of DNA hypermethylation could expand prospects for the development of early diagnostic biomarkers.

\section{The Inactivation of Tumor Suppressor Genes on Chromosome 3}

The current genomic changes in NPC detected by genome-wide approaches such as allelotype studies and comparative genomic hybridization have identified high frequencies of genetic imbalances on various chromosomes such as 1q, 2q, 3p, 9p, 11p, 12q, 13q, 14q, 16q, and 17q [11-14]. Among the chromosome abnormalities, the silencing of TSGs on chromosome 3 is a frequent event in nasopharyngeal tumorigenesis [13,15-17]. These findings suggest that inactivation of TSGs on chromosome 3 is an early and crucial event in NPC development and progress [13]. In this review, we summarize the function of TSG on chromosome 3 to understand the role of TSGs on chromosome 3 in the development of NPC, suggested to be a promising target for early diagnostic biomarkers. The most important epigenetic event is the hypermethylation at multiple chromosome 3p TSG. The silencing of TSGs on chromosome 3p, including ADAM, BLU, DLEC1, GNAT1, LARS2, LTF, MLH1, RASSF1A, RAR- $\beta$, TIG1, VLH1, PTPRG, and ITGA9, by the phenomenon of DNA aberrant hypermethylation in NPC, seems to be the key role in the mechanism of NPC tumorigenesis, as reported. Roles of TSGs on chromosome 3 during nasopharyngeal carcinogenesis, including cell proliferation, cell cycle regulation, apoptosis, etc., have been reported in recent years (Table 1 ).

Table 1. Roles of TSGs on chromosome 3 during nasopharyngeal carcinogenesis.

\begin{tabular}{cccc}
\hline Gene & Location & Roles & References \\
\hline ADAMTS9 & $3 \mathrm{p} 14.1$ & Angiogenesis & {$[6]$} \\
\hline PTPRG & $3 \mathrm{p} 14.2$ & Chromosomal translocations and deletions, \\
cell cycle & {$[18]$} \\
\hline BLU & $3 \mathrm{p} 21.3$ & Cell progress, stress-response & {$[7,16,17,19,20]$} \\
\hline GNAT1 & $3 \mathrm{p} 21.3$ & Remains to be revealed & {$[21]$} \\
\hline LARS2 & $3 \mathrm{p} 21.3$ & Protein synthesis & {$[22]$} \\
\hline LTF & $3 \mathrm{p} 21.3$ & $\begin{array}{c}\text { Immunomodulatory, homeostasis, anti-tumor } \\
\text { activity, cell growth, cell cycle regulatory }\end{array}$ & {$[23-25]$} \\
\hline MLH1 & $3 \mathrm{p} 21.3$ & Mismatch repair & {$[26,27]$} \\
\hline RASSF1A & $3 \mathrm{p} 21.3$ & $\begin{array}{c}\text { Cell proliferation, cell cycle regulation, } \\
\text { apoptosis, micro-tubular stabilization }\end{array}$ & {$[5,13,26-38]$} \\
\hline ITGA9 & $3 \mathrm{p} 21.3$ & Cell-cell and cell-matrix adhesion & {$[15]$} \\
\hline DLEC1 & $3 \mathrm{p} 22.2$ & $\begin{array}{c}\text { Cell communication, signaling transduction, } \\
\text { cell proliferation }\end{array}$ & {$[7,38-40]$} \\
\hline RAR- $\beta$ & $3 \mathrm{p} 24.2$ & $\begin{array}{c}\text { Hormone receptor, transcriptional regulator, } \\
\text { retinoic acid signaling, cell growth } \\
\text { and differentiation }\end{array}$ & {$[28,30,36]$} \\
\hline VLH1 & $3 \mathrm{p} 25.3$ & Ubiquitination & {$[27]$} \\
\hline TIG1 & $3 \mathrm{q} 25.3$ & Cell-to-cell contact & {$[39,41]$} \\
\hline
\end{tabular}


Among regions belonging to 3p, multiple genes located at the region of 3p21.3 have been widely studied in NPC xenografts and cell lines and in primary tumors. Based on previous studies, shown in Table 1, RASSF1A was reported to be the most studied TSG involved in the tumorigenesis of nasopharynx.

\section{RASSF1A and Panel of Genes as Methylation Biomarkers for NPC}

Ras Association domain Family 1A (RASSF1A) gene, located on chromosome 3p21.3, was first mapped by Dammann et al. (2000). They reported that the inactivated expression of RASSF1A transcript was frequently observed in lung cancer by the mechanism of hypermethylation [42]. The inactivation of $R A S S F 1 A$ has been identified as one of the early events that drives to nasopharyngeal carcinoma (Figure 1) [3,43]. It was reported that RASSF1A induces cell-cycle arrest in the phase of G0/G1 by inhibiting the accumulation of cyclin D1; thus, RASSF1A blocks the cell cycle progression. RASSF1A is suggested to be an important human tumor suppressor protein acting at the level of G1/S-phase cell cycle progression [44]. Later on, a significant correlation between the methylation status of RASSF1A with NPC was reported in the NPC cell lines, xenografts, and primary NPC tumors collected from Asian patients, but not in the normal nasopharyngeal epithelia [34]. In their report, they investigated the finding that the 5' CpG of RASSF1A's promoter hypermethylation was detected in 75\% of cell lines (C666-1, CNE-1, and CNE-2), $100 \%$ of xenografts (xeno-2117, xeno-1915, xeno-8, and xeno-666), and $66.67 \%$ of primary tumors. Specifically, no RASSF1A expression was detected on cell lines as well as xenografts. The re-expression of RASSF1A in cell lines was detected after treatment with 5'-aza-2'deoxycytidine [34]. In the study of Wang et al. (2009), the expression of RASSF1A was downregulated in the cell line of CNE-2 due to promoter hypermethylation. The loss of RASSF1A expression was greatly restored by the treatment of 5'-aza-2'deoxycytidine [33]. Again, according to the systematic reviews and meta-analyses by Ye et al. (2017), they concluded that methylation of RASSF1A promoter may be associated with the development, progression, and metastasis of NPC through the evaluation of the pooled sensitivity, specificity, and AUC of RASSF1A promoter methylation in NPC samples vs. non-tumor samples [45]. Additionally, hypermethylation of RASSF1A was reported as a frequent and significant event in EBV-positive NPC, and may be an important event in the pathogenesis of EBV-infected NPC. Moreover, the hypermethylation of $R A S S F 1 A$ could serve as a potential biomarker for worse overall survival [46]. Based on these results, promoter hypermethylation of $R A S S F 1 A$, leading to transcriptional inactivation, acts as the critical target gene involved in nasopharyngeal tumorigenesis. Further functional evidence supporting RASSF1A as the potential candidate for the NPC targeting strategy was demonstrated in the in vivo BALB/c nude mice assay. In their report, tumors were dramatically reduced in the RASSF1A-tranfected nude mice [13]. Taken together, these findings suggest that RASSF1A is the best candidate tumor suppressor at 3p21.3 in NPC.

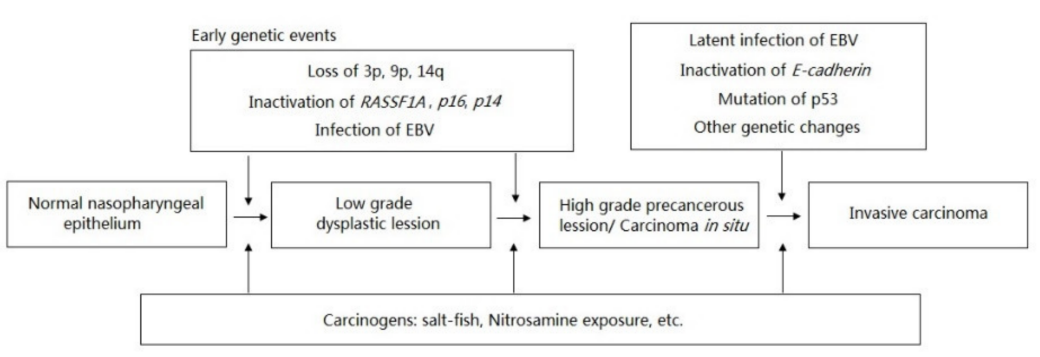

Figure 1. Inactivation of RASSF1A—the early genetic event driving NPC.

The specificity, sensitivity, positive predictive value, and negative predictive value of methylated RASSF1A-based diagnosis vary from $87.14 \%$ to $100.00 \%, 0.00 \%$ to $91.18 \%, 0.00 \%$ to $100.00 \%$, and $25.00 \%$ to $72.62 \%$, respectively (Table 2 ). The low sensitivity and specificity of a single-gene biomarker limits the use of one gene for cancer diagnosis. Therefore, 
for the aim of increasing the diagnosis power in NPC, DNA methylation of a panel of different genes combined with RASSF1A was analyzed. Given the evidence of methylation recorded in NPC, a panel of other useful methylated biomarkers for NPC is summarized in Table 3. Taking into account that at least one gene was methylated, gains in specificity, sensitivity, positive predictive value, and negative predictive value of diagnosis, compared to corresponding single-gene RASSF1A, were recorded. When the methylation of at least one gene was considered, all values could increase to a value of $100 \%$. Kwong et al. (2002) examined the methylation of eight genes, including RASSF1A, RAR- $\beta, D A P K, p 16, p 15$, $p 14, M G M T$, and GSTP1, in primary tumors and normal epithelium. They reported that no methylation of these genes was detected in the six normal nasopharyngeal epithelium samples. In the case of primary tumors, only one gene of GSTP1 was unmethylated. They found that aberrant methylation in at least one of the seven genes, including RASSF1A, $R A R-\beta, D A P K, p 16, p 15, p 14$, and MGMT, was detectable in $100 \%$ of the tumors of patients; specificity, sensitivity, positive predictive value, and negative predictive value reached $100 \%$ for each value [30].

Table 2. Specificity, sensitivity, positive predictive value, and negative predictive value of methylated RASSF1A-based diagnosis (focused on the case-control study).

\begin{tabular}{|c|c|c|c|c|c|}
\hline $\begin{array}{l}\text { NPC Case } \\
\quad(n)\end{array}$ & $\begin{array}{l}\text { Non-Cancerous Control } \\
\text { (n) }\end{array}$ & Case & Control & $\mathrm{Sp}, \mathrm{Se}, \mathrm{Po}, \mathrm{Ne}$ & References \\
\hline $\begin{array}{l}\text { Primary tumor tissue } \\
\text { (28) }\end{array}$ & $\begin{array}{c}\text { Nasopharyngeal epithelium } \\
(6)\end{array}$ & $\begin{array}{c}14 / 21 \\
(66.67 \%)\end{array}$ & $\begin{array}{c}0 / 6 \\
(0.00 \%)\end{array}$ & $\begin{array}{c}\mathrm{Sp}=100.00 \% \\
\mathrm{Se}=66.67 \% \\
\mathrm{Po}=100.00 \% \\
\mathrm{Ne}=46.15 \%\end{array}$ & [34] \\
\hline $\begin{array}{l}\text { Primary tumor tissue } \\
\text { (28) }\end{array}$ & $\begin{array}{l}\text { Tissue } \\
(6)\end{array}$ & $\begin{array}{c}23 / 28 \\
(82.14 \%)\end{array}$ & $\begin{array}{c}0 / 6 \\
(0.00 \%)\end{array}$ & $\begin{array}{c}\mathrm{Sp}=100.00 \% \\
\mathrm{Se}=82.14 \% \\
\mathrm{Po}=100.00 \% \\
\mathrm{Ne}=54.55 \%\end{array}$ & {$[30]$} \\
\hline $\begin{array}{l}\text { Nasopharyngeal brushing } \\
\text { (28) }\end{array}$ & $\begin{array}{c}\text { Nasopharyngeal brushing } \\
\text { (12) }\end{array}$ & $\begin{array}{c}11 / 28 \\
(39.29 \%)\end{array}$ & $\begin{array}{c}0 / 26 \\
(0.00 \%)\end{array}$ & $\begin{array}{c}\mathrm{Sp}=100.00 \% \\
\mathrm{Se}=39.29 \% \\
\mathrm{Po}=100.00 \% \\
\mathrm{Ne}=60.47 \%\end{array}$ & [31] \\
\hline $\begin{array}{l}\text { Primary tumor tissue } \\
\text { (30) }\end{array}$ & $\begin{array}{l}\text { Tissue } \\
(6)\end{array}$ & $\begin{array}{c}20 / 30 \\
(66.67 \%)\end{array}$ & $\begin{array}{c}0 / 6 \\
(0.00 \%)\end{array}$ & $\begin{array}{c}\mathrm{Sp}=100.00 \% \\
\mathrm{Se}=66.67 \% \\
\mathrm{Po}=100.00 \% \\
\mathrm{Ne}=37.50 \%\end{array}$ & \\
\hline $\begin{array}{c}\text { Nasopharyngeal brushing } \\
\text { (30) }\end{array}$ & $\begin{array}{c}\text { Nasopharyngeal brushing } \\
\text { (37) }\end{array}$ & $\begin{array}{c}10 / 30 \\
(33.33 \%)\end{array}$ & $\begin{array}{c}0 / 37 \\
(0.00 \%)\end{array}$ & $\begin{array}{c}\mathrm{Sp}=100.00 \% \\
\mathrm{Se}=33.33 \% \\
\mathrm{Po}=100.00 \% \\
\mathrm{Ne}=64.91 \%\end{array}$ & \\
\hline $\begin{array}{l}\text { M\&T rinsing fluid } \\
\text { (30) }\end{array}$ & M\&T rinsing fluid & $\begin{array}{c}11 / 30 \\
(36.67 \%)\end{array}$ & $\begin{array}{c}0 / 43 \\
(0.00 \%)\end{array}$ & $\begin{array}{c}\mathrm{Sp}=100.00 \% \\
\mathrm{Se}=36.67 \% \\
\mathrm{Po}=100.00 \% \\
\mathrm{Ne}=69.36 \%\end{array}$ & [29] \\
\hline $\begin{array}{l}\text { Plasma } \\
\text { (30) }\end{array}$ & $\begin{array}{l}\text { Plasma } \\
\text { (43) }\end{array}$ & $\begin{array}{c}9 / 30 \\
(30.00 \%)\end{array}$ & $\begin{array}{c}1 / 43 \\
(2.32 \%)\end{array}$ & $\begin{array}{l}\mathrm{Sp}=97.67 \% \\
\mathrm{Se}=30.00 \% \\
\mathrm{Po}=90.00 \% \\
\mathrm{Ne}=66.67 \%\end{array}$ & \\
\hline $\begin{array}{l}\text { Buffy coat } \\
\text { (30) }\end{array}$ & $\begin{array}{l}\text { Buffy coat } \\
\quad(43)\end{array}$ & $\begin{array}{c}0 / 30 \\
(0.00 \%)\end{array}$ & $\begin{array}{c}1 / 43 \\
(2.32 \%)\end{array}$ & $\begin{array}{c}\mathrm{Sp}=97.67 \% \\
\mathrm{Se}=0.00 \% \\
\mathrm{Po}=0.00 \% \\
\mathrm{Ne}=58.33 \%\end{array}$ & \\
\hline
\end{tabular}


Table 2. Cont.

\begin{tabular}{|c|c|c|c|c|c|}
\hline $\begin{array}{l}\text { NPC Case } \\
(\mathrm{n})\end{array}$ & $\begin{array}{c}\text { Non-Cancerous Control } \\
\text { (n) }\end{array}$ & Case & Control & $\mathrm{Sp}, \mathrm{Se}, \mathrm{Po}, \mathrm{Ne}$ & References \\
\hline $\begin{array}{l}\text { Tumor tissue } \\
\text { (28) }\end{array}$ & $\begin{array}{l}\text { Tissue } \\
(5)\end{array}$ & $\begin{array}{c}13 / 28 \\
(46.43 \%)\end{array}$ & $\begin{array}{c}0 / 5 \\
(0.00 \%)\end{array}$ & $\begin{array}{c}\mathrm{Sp}=100.00 \% \\
\mathrm{Se}=46.43 \% \\
\mathrm{Po}=100.00 \% \\
\mathrm{Ne}=25.00 \%\end{array}$ & [27] \\
\hline $\begin{array}{l}\text { Plasma } \\
\qquad(41)\end{array}$ & $\begin{array}{l}\text { Plasma } \\
\text { (43) }\end{array}$ & $\begin{array}{c}2 / 41 \\
(4.88 \%)\end{array}$ & $\begin{array}{c}0 / 43 \\
(0.00 \%)\end{array}$ & $\begin{array}{c}\mathrm{Sp}=100.00 \% \\
\mathrm{Se}=4.88 \% \\
\mathrm{Po}=100.00 \% \\
\mathrm{Ne}=52.44 \%\end{array}$ & [26] \\
\hline $\begin{array}{l}\text { Tumor tissue } \\
(68)\end{array}$ & $\begin{array}{l}\text { Tissue } \\
(9)\end{array}$ & $\begin{array}{c}62 / 68 \\
(91.18 \%)\end{array}$ & $\begin{array}{c}0 / 9 \\
(0.00 \%)\end{array}$ & $\begin{array}{c}\mathrm{Sp}=100.00 \% \\
\mathrm{Se}=91.18 \% \\
\mathrm{Po}=100.00 \% \\
\mathrm{Ne}=60.00 \%\end{array}$ & [36] \\
\hline $\begin{array}{l}\text { Tumor tissue } \\
\qquad(38)\end{array}$ & $\begin{array}{l}\text { Tissue } \\
(14)\end{array}$ & $\begin{array}{c}27 / 38 \\
(71.05 \%)\end{array}$ & $\begin{array}{c}0 / 14 \\
(0.00 \%)\end{array}$ & $\begin{array}{c}\mathrm{Sp}=100.00 \% \\
\mathrm{Se}=71.05 \% \\
\mathrm{Po}=100.00 \% \\
\mathrm{Ne}=56.00 \%\end{array}$ & [33] \\
\hline $\begin{array}{l}\text { Tumor tissue/brushing } \\
\text { (53) }\end{array}$ & $\begin{array}{c}\text { Nasopharyngeal brushing } \\
(25)\end{array}$ & $\begin{array}{c}40 / 53 \\
(75.47 \%)\end{array}$ & $\begin{array}{c}1 / 25 \\
(4.00 \%)\end{array}$ & $\begin{array}{l}\mathrm{Sp}=96.00 \% \\
\mathrm{Se}=45.47 \% \\
\mathrm{Po}=97.56 \% \\
\mathrm{Ne}=64.87 \%\end{array}$ & [5] \\
\hline $\begin{array}{l}\text { Tumor tissue } \\
\text { (36) }\end{array}$ & $\begin{array}{c}\text { Tissue } \\
(19)\end{array}$ & $\begin{array}{c}27 / 36 \\
(75.00 \%)\end{array}$ & $\begin{array}{c}0 / 19 \\
(0.00 \%)\end{array}$ & $\begin{array}{c}\mathrm{Sp}=100.00 \% \\
\mathrm{Se}=75.00 \% \\
\mathrm{Po}=100.00 \% \\
\mathrm{Ne}=67.86 \%\end{array}$ & [28] \\
\hline $\begin{array}{l}\text { Tumor tissue } \\
(49)\end{array}$ & $\begin{array}{l}\text { Tissue } \\
(20)\end{array}$ & $\begin{array}{c}39 / 49 \\
(79.59 \%)\end{array}$ & $\begin{array}{c}0 / 20 \\
(0.00 \%)\end{array}$ & $\begin{array}{c}\mathrm{Sp}=100.00 \% \\
\mathrm{Se}=79.59 \% \\
\mathrm{Po}=100.00 \% \\
\mathrm{Ne}=66.67 \%\end{array}$ & [201 \\
\hline $\begin{array}{l}\text { Nasopharyngeal brushing } \\
(49)\end{array}$ & $\begin{array}{c}\text { Nasopharyngeal brushing } \\
(20)\end{array}$ & $\begin{array}{c}29 / 49 \\
(59.18 \%)\end{array}$ & $\begin{array}{c}0 / 20 \\
(0.00 \%)\end{array}$ & $\begin{array}{c}\mathrm{Sp}=100.00 \% \\
\mathrm{Se}=59.18 \% \\
\mathrm{Po}=100.00 \% \\
\mathrm{Ne}=50.00 \%\end{array}$ & {$[32]$} \\
\hline $\begin{array}{l}\text { Serum } \\
(40)\end{array}$ & $\begin{array}{l}\text { Serum } \\
(41)\end{array}$ & $\begin{array}{c}7 / 40 \\
(17.50 \%)\end{array}$ & $\begin{array}{c}2 / 41 \\
(4.87 \%)\end{array}$ & $\begin{array}{l}\mathrm{Sp}=95.12 \% \\
\mathrm{Se}=17.50 \% \\
\mathrm{Po}=77.78 \% \\
\mathrm{Ne}=54.17 \%\end{array}$ & [38] \\
\hline $\begin{array}{l}\text { Tumor tissue } \\
\text { (70) }\end{array}$ & $\begin{array}{c}\text { Nasopharyngeal brushing } \\
(70)\end{array}$ & $\begin{array}{c}47 / 70 \\
(52.22 \%)\end{array}$ & $\begin{array}{c}9 / 70 \\
(12.86 \%)\end{array}$ & $\begin{aligned} \mathrm{Sp} & =87.14 \% \\
\mathrm{Se} & =67.14 \% \\
\mathrm{Po} & =83.93 \% \\
\mathrm{Ne} & =72.62 \%\end{aligned}$ & [35] \\
\hline
\end{tabular}

Note: Sp: specificity, Se: sensitivity; Po: positive predictive value; Ne: negative predictive value; M\&T rinsing fluid: mouth and throat rinsing fluid.

Table 3. RASSF1A combined with other useful methylated biomarkers for NPC (focused on the case-control study).

\begin{tabular}{|c|c|c|c|c|c|}
\hline Panel of Genes & $\begin{array}{l}\text { NPC Case } \\
\text { (n) }\end{array}$ & $\begin{array}{l}\text { Non-Cancerous Control } \\
(\mathrm{n})\end{array}$ & MI & $\mathrm{Sp}, \mathrm{Se}, \mathrm{Po}, \mathrm{Ne}$ & References \\
\hline $\begin{array}{c}\text { RASSF1A, RAR- } \beta, D A P K \\
p 16, p 15, p 14, \\
M G M T, G S T P 1\end{array}$ & $\begin{array}{l}\text { Primary tumor } \\
\text { tissue } \\
(28)\end{array}$ & $\begin{array}{l}\text { Nasopharyngeal } \\
\text { epithelium } \\
\text { (6) }\end{array}$ & $\begin{array}{l}\text { 28/28 }(100.00 \%) \text { of cases, at } \\
\text { least one of seven genes: } \\
\text { RASSF1A, RAR- } \beta, D A P K, p 16, \\
p 15, p 14, \text { and } M G M T . \\
\text { All controls were not } \\
\text { unmethylated. }\end{array}$ & $\begin{array}{l}\mathrm{Sp}=100.00 \% \\
\mathrm{Se}=100.00 \% \\
\mathrm{Po}=100.00 \% \\
\mathrm{Ne}=100.00 \%\end{array}$ & [30] \\
\hline
\end{tabular}


Table 3. Cont.

\begin{tabular}{|c|c|c|c|c|c|}
\hline Panel of Genes & $\begin{array}{l}\text { NPC Case } \\
\quad(n)\end{array}$ & $\begin{array}{l}\text { Non-Cancerous Control } \\
\text { (n) }\end{array}$ & MI & $\mathrm{Sp}, \mathrm{Se}, \mathrm{Po}, \mathrm{Ne}$ & References \\
\hline RASSF1A, DAPK, p16 & $\begin{array}{l}\text { Nasopharyngeal } \\
\text { brushing } \\
\text { (28) }\end{array}$ & $\begin{array}{l}\text { Nasopharyngeal } \\
\text { brushing } \\
\text { (12) }\end{array}$ & $\begin{array}{c}22 / 28(78.57 \%) \text { of cases, at least } \\
\text { one of three genes. } \\
\text { All controls were not } \\
\text { unmethylated. }\end{array}$ & $\begin{array}{l}\mathrm{Sp}=78.57 \% \\
\mathrm{Se}=100.00 \% \\
\mathrm{Po}=100.00 \% \\
\mathrm{Ne}=66.67 \%\end{array}$ & [31] \\
\hline \multirow{5}{*}{$\begin{array}{l}\text { RASSF1A, E-cadherin, } \\
\text { DAPK, p15, p16 }\end{array}$} & $\begin{array}{l}\text { Primary tumor } \\
\text { tissue } \\
\text { (30) }\end{array}$ & $\begin{array}{l}\text { Tissue } \\
(6)\end{array}$ & $\begin{array}{c}29 / 30 \text { of cases, at least one of } \\
\text { three genes. } \\
\text { All controls were not } \\
\text { unmethylated. }\end{array}$ & $\begin{array}{c}\mathrm{Sp}=100.00 \% \\
\mathrm{Se}=96.97 \% \\
\mathrm{Po}=100.00 \% \\
\mathrm{Ne}=85.71 \%\end{array}$ & \multirow{5}{*}{ [29] } \\
\hline & $\begin{array}{l}\text { Nasopharyngeal } \\
\text { brushing } \\
\text { (30) }\end{array}$ & $\begin{array}{l}\text { Nasopharyngeal } \\
\text { brushing } \\
\text { (37) }\end{array}$ & $\begin{array}{c}24 / 30(80.00 \%) \text { of cases, at least } \\
\text { one of three genes. } \\
\text { All controls were not } \\
\text { unmethylated. }\end{array}$ & $\begin{array}{c}\mathrm{Sp}=100.00 \% \\
\mathrm{Se}=80.00 \% \\
\mathrm{Po}=100.00 \% \\
\mathrm{Ne}=86.05 \%\end{array}$ & \\
\hline & $\begin{array}{l}\text { M\&T rinsing fluid } \\
(30)\end{array}$ & M\&T rinsing fluid & $\begin{array}{c}26 / 30(87.00 \%) \text { of cases, at least } \\
\text { one of three genes. } \\
1 / 43(2.32 \%) \text { of controls, at least } \\
\text { one of three genes. }\end{array}$ & $\begin{array}{l}\mathrm{Sp}=97.67 \% \\
\mathrm{Se}=86.67 \% \\
\mathrm{Po}=96.30 \% \\
\mathrm{Ne}=91.30 \%\end{array}$ & \\
\hline & $\begin{array}{l}\text { Plasma } \\
(30)\end{array}$ & $\begin{array}{l}\text { Plasma } \\
(43)\end{array}$ & $\begin{array}{l}3 / 30(10.00 \%) \text { of cases, at least } \\
\text { one of three genes. } \\
2 / 43(4.65 \%) \text { of controls, at least } \\
\text { one of three genes. }\end{array}$ & $\begin{array}{l}\mathrm{Sp}=95.34 \% \\
\mathrm{Se}=10.00 \% \\
\mathrm{Po}=60.00 \% \\
\mathrm{Ne}=60.30 \%\end{array}$ & \\
\hline & $\begin{array}{l}\text { Buffy coat } \\
(30)\end{array}$ & $\begin{array}{l}\text { Buffy coat } \\
\quad(43)\end{array}$ & $\begin{array}{c}12 / 30(40.00 \%) \text { of cases, at least } \\
\text { one of three genes. } \\
3 / 43(6.97 \%) \text { of controls, at least } \\
\text { one of three genes. }\end{array}$ & $\begin{array}{l}\mathrm{Sp}=93.02 \% \\
\mathrm{Se}=40.00 \% \\
\mathrm{Po}=80.00 \% \\
\mathrm{Ne}=68.97 \%\end{array}$ & \\
\hline $\begin{array}{c}\text { RASSF1A, MLH1, CDH1, } \\
\text { CDK2B, THBS1, MGMT, } \\
\text { CDKN2A, TP73, C8, } \\
\text { ARF, VHL }\end{array}$ & $\begin{array}{l}\text { Tumor tissue } \\
\text { (28) }\end{array}$ & $\begin{array}{l}\text { Tissue } \\
(5)\end{array}$ & $\begin{array}{c}\text { 26/28 (92.86\%) of cases, at least } \\
\text { one of ten genes: RASSF1A, } \\
\text { MLH1, CDH1, CDK2B, THBS1, } \\
\text { MGMT, CDKN2A, TP73, C8, } \\
\text { and ARF. } \\
\text { All controls were not } \\
\text { unmethylated. }\end{array}$ & $\begin{array}{c}\mathrm{Sp}=100.00 \% \\
\mathrm{Se}=92.86 \% \\
\mathrm{Po}=100.00 \% \\
\mathrm{Ne}=71.43 \%\end{array}$ & [27] \\
\hline $\begin{array}{c}\text { RASSF1A, MLH1, CDH1, } \\
\text { DAPK, p15, p16 }\end{array}$ & $\begin{array}{l}\text { Plasma } \\
(41)\end{array}$ & $\begin{array}{l}\text { Plasma } \\
(43)\end{array}$ & $\begin{array}{l}29 / 41(70.73 \%) \text { of cases, at least } \\
\text { one of six genes. } \\
4 / 43(9.30 \%) \text { of controls, at least } \\
\text { one of six genes. }\end{array}$ & $\begin{array}{l}\mathrm{Sp}=90.70 \% \\
\mathrm{Se}=70.73 \% \\
\mathrm{Po}=87.88 \% \\
\mathrm{Ne}=76.47 \%\end{array}$ & [26] \\
\hline$R A S S F 1 A, R A R-\beta, D A P K$ & $\begin{array}{l}\text { Tumor tissue } \\
(68)\end{array}$ & $\begin{array}{l}\text { Tissue } \\
(9)\end{array}$ & $\begin{array}{c}67 / 68(98.53 \%) \text { of cases, at least } \\
\text { one of three genes. } \\
\text { All controls were not } \\
\text { unmethylated. }\end{array}$ & $\begin{array}{c}\mathrm{Sp}=100.00 \% \\
\mathrm{Se}=91.18 \% \\
\mathrm{Po}=100.00 \% \\
\mathrm{Ne}=60.00 \%\end{array}$ & [36] \\
\hline $\begin{array}{c}\text { RASSF1A, CHFR, RIZ1, } \\
\text { WIFI1, p16, RASSF2A, } \\
\text { DAPK1, DLC1, } \\
\text { CDH13, CADM1 }\end{array}$ & $\begin{array}{c}\text { Tumor } \\
\text { tissue/brushing } \\
\text { (53) }\end{array}$ & $\begin{array}{l}\text { Nasopharyngeal } \\
\text { brushing } \\
\text { (25) }\end{array}$ & $\begin{array}{c}52 / 53(98.11 \%) \text { of cases, at least } \\
\text { one of ten genes. } \\
\text { nc in controls. }\end{array}$ & nc & [5] \\
\hline $\begin{array}{l}\text { RASSF1A, RAR- } \beta, \text { SHP1, } \\
\text { DAPK, } 1616, \text { GSTP1, TIMP3, } \\
\text { APC, CDH1, MGMT }\end{array}$ & $\begin{array}{l}\text { Tumor tissue } \\
\text { (36) }\end{array}$ & $\begin{array}{l}\text { Tissue } \\
(19)\end{array}$ & nc & nc & [28] \\
\hline \multirow[b]{2}{*}{ RASSF1A, DAPK } & $\begin{array}{l}\text { Tumor tissue } \\
\text { (49) }\end{array}$ & $\begin{array}{l}\text { Tissue } \\
(20)\end{array}$ & $\mathrm{nc}$ & nc & \multirow[b]{2}{*}{ [32] } \\
\hline & $\begin{array}{l}\text { Nasopharyngeal } \\
\text { brushing } \\
\text { (49) }\end{array}$ & $\begin{array}{l}\text { Nasopharyngeal } \\
\text { brushing } \\
\text { (20) }\end{array}$ & nc & nc & \\
\hline $\begin{array}{c}\text { RASSF1A, DLEC1, } \\
\text { CDKN2A, DAPK, UCHL1 }\end{array}$ & $\begin{array}{l}\text { Serum } \\
(40)\end{array}$ & $\begin{array}{l}\text { Serum } \\
(41)\end{array}$ & $\begin{array}{c}\text { 34/40 (85.00\%) of cases, at least } \\
\text { one of five genes. } \\
15 / 41(36.59 \%) \text { of controls, at } \\
\text { least one of five genes. }\end{array}$ & $\begin{array}{l}\mathrm{Sp}=85.00 \% \\
\mathrm{Se}=63.42 \% \\
\mathrm{Po}=69.39 \% \\
\mathrm{Ne}=81.25 \%\end{array}$ & [38] \\
\hline
\end{tabular}

Note: MI: methylation index; Sp: specificity; Se: sensitivity; Po: positive predictive value; Ne: negative predictive value; M\&T rinsing fluid: mouth and throat rinsing fluid, nc: no record/non-calculation. Genes in bold were located on 3p. 
According to the World Health Organization, pathological evidence found by biopsies is the gold standard for NPC diagnosis [47]. Biopsy-based diagnosis faces difficulty in that the symptoms of NPC are not conclusive at early stages. Additionally, almost patients were diagnosed at the advanced stage. Therefore, searching the minimally invasive biomarkers for the early stage, as well as monitoring the disease progression, is an urgent need. A variety of non-invasive or minimally invasive sampling methods have been attempted for the development of the non-invasive diagnosis of NPC. A panel of methylation markers consisting of RASSF1A and other TSGs has been proposed as the complementary test for diagnosis of NPC based on the minimal invasive or non-invasive samples, including plasma, M\&T rinsing fluid, buffy coat, and nasopharyngeal brushing $[5,26,29,31,32]$. For example, in the study of Chang et al. (2003), they compared the power of methylation markers on different sources of samples; as a result, the panel of five methylation markers including RASSF1A, E-cadherin, DAPK, $p 15$, and $p 16$ had a specificity of $100.00 \%$ and sensitivity of $80.00 \%$ [29]. Hutajulu et al. (2011) reported that a panel of methylation biomarkers consisting of RASSF1A, CHFR, $p 16$, RIZ1, and WIF1 showed high frequency of at least one methylated gene of NPC compared to individual markers, providing good discrimination between NPC and non-cancer [5].

Circulating cell-free Epstein-Barr virus (EBV) levels have emerged as a promising biomarker in clinical decision making and improving NPC treatment $[28,31,37,48]$. It could be explained that EBV DNA load has been implied as the factor that contributes the methylation alterations through the activation of DNA methyltransferase by EBV LMP-1, which involves c-JUN NH (2)-terminal kinase signaling or interaction with transcriptional repression [49,50]. Hence, analysis of methylation status of selected TSGs is proposed as the additive test to EBV-DNA detected assay for early diagnosis of NPC [5,51]. Zhou et al. (2005) reported that higher frequency of RASSF1A methylation and higher viral load were detected in the $\mathrm{T}$ tissue (containing more than $70 \%$ of tumor cells) compared to $\mathrm{P}$ and $\mathrm{Z}$ tissues (located 0.5 and $1.0 \mathrm{~cm}$ outside of visible NPC lesions, respectively). They concluded that hypermethylation of RASSF1A and high EBV load might be important events in NPC pathogenesis, and they may be useful molecular diagnostic markers for NPC [37]. The method of multiplex methylation-specific PCR, which could simultaneously detect methylation of RASSF1A and DAPK and the presence of EBV DNA (EBNA-1 and $L M P 1)$, using only picograms of tumor DNA from NP swabs, has been developed. The specificity and sensitivity of this method are $100 \%$ and $98 \%$, respectively, in detecting 49 NPCs which include 19 early-stage patients [32]. Therefore, these studies opened the way to develop innovative diagnosis strategies for NPC.

The last decades have seen the development of a plethora of drugs that were designed to specifically target the phenomenon of DNA aberrant hypermethylation, which was identified as the driver of cancer development, including NPC. To date, two nucleoside analogues, including 5-azacytidine and 5'-aza-2'deoxycytidine, have been approved as therapeutic agents for cancers [52]. Mechanically, these drugs, when incorporated into DNA, produce a covalent DNMT-DNA complex, resulting in inactivated DNMTs by the subsequent degradation of the trapped enzymes, also initiating DNA damage [53]. The data of in vitro and in vivo indicate that 5-azacytidine, and 5'-aza-2'deoxycytidine greatly restored the expression of RASSF1A. For instance, the NPC cell line C666-1, which was confirmed to be completely methylated and with no expression of RASSF1A, was administrated with 5'-aza-2'deoxycytidine. The restoration of RASSF1A was observed in the 5'-aza-2'deoxycytidine-treated cell line [34]. The treatment with 5-azacytidine, as reported, could enhance the radiosensitivity of both the CNE2 and SUNE1 NPC cell lines. Notably, the combination of both 5-azacytidine treatment and irradiation significantly inhibited the growth of the tumor in the mouse xenograft model and enhanced radiationinduced apoptosis in vitro compared to 5-azaC alone or IR alone [54].

Moreover, the determination of RASSF1A downstream effectors may provide potential interest for therapeutic regulators in patients with NPC, especially metastatic NPC [55]. According to their study, they reported that the expression of RASSF1A inactivates YAP1 by 
remodeling F-actin assembly, as the results, suppress the transcriptional activity of PDGFB, which was reported as the important element for sustaining the malignant phenotypes of NPC cells. The silence of PDGFB abrogated the RASSF1A depletion-induced malignant phenotypes of NPC cells [55]. Thus, it could be inferred that the inactivation of RASSF1A, mechanistically, may, through the epigenetic event of hypermethylation, trigger the process of NPC. Moreover, it is suggested that RASSF1A inhibits malignant phenotypes by repressing PDGFB expression in a YAP1-dependent manner and PDGFB could serve as the potential therapeutic agent for metastatic NPC treatment [55].

\section{Other Genes Located at 3p Have Also Been Studied though Not as much as RASSF1A}

Besides RASSF1A, other genes, including Blu, RAR- $\beta, D L E C 1$, and LTF have also been studied. A recent report indicated that $B l u$ is a stress-responsive gene and is regulated by heat shock and environmental factors. Moreover, the overexpression of Blu was reported to inhibit the colony formation of cancer cells [16]. The first evidence of downregulated Blu by the aberrant hypermethylation involved in the pathogenesis of NPC was reported by Liu et al. (2003). They found high frequency of promoter hypermethylation in $74 \%$ (17 of 23) of primary tumors and $100 \%$ of cell lines, including CNE1, CNE2, and HNE1, in contrast to the status in which no methylation was shown in non-cancerous controls. The restoration of Blu expression was observed in CNE2 by the treatment of 5'-aza-2'deoxycytidine [19]. The aberrant hypermethylation of Blu was reported in Tunisian NPC patients. The frequency of Blu was $34.1 \%$ and $86.3 \%$, respectively [7]. The frequency of methylation Blu in Tunisian NPC patients was lower than in Chinese NPC patients, in which it was 66\% (19 of 29 primary tumors) and Vietnamese NPC patients, in which it was $81.11 \%$ (73 of 90 NPC tissues) $[7,17,20]$. It is noted that there was a significant correlation between aberrant methylation of the Blu promoter and undifferentiated histological type of NPC [7]. Thus, the aberrant methylation of Blu is the common event in the development of NPC.

The aberrant hypermethylation of DLEC1 was also reported in Tunisian NPC patients with a frequency of $86.3 \%$ [7]. The recorded methylation frequency of DLEC1 in their report was higher than the frequency of $71.43 \%$ (30 of 42 NPC primary tumors) in Chinese NPC patients and the frequency of $60.42 \%$ (29 of 48 NPC tissues) in NPC patients of Hong Kong [7,39,40]. Only the single-gene DLEC1-based method had a specificity of $96 \%$ and sensitivity of $60 \%$ in diagnosis of NPC [39]. Sensitivity reached $78 \%$ and specificity was unchanged in the case of combining DLEC1 and KIF1A [39]. Similar to previous studies, Tian et al. (2013) also investigated promoter methylation of DLEC1, as well as combination with RASSF1A, CDKN2A,DAPK, and UCHL1 as the methylation markers in the diagnosis of NPC in serum samples [38]. Thus, screening DNA hypermethylation of DLEC1 in serum is a promising strategy for the diagnosis of NPC.

$R A R-\beta$ belongs to the nuclear receptor superfamily and it mediates cellular signaling, cell growth, and differentiation. Kwong et al. (2002) reported the aberrant methylation of $R A R-\beta$ in NPC primary tumors, cell lines, and xenograft. Aberrant methylation was significantly observed in $81 \%$ (32 of 40 cases) of primary tumor, $75 \%$ ( 3 of 4 cases) of cell lines, and 50\% (2 of 4 cases) of xenografts, compared to normal nasopharyngeal epithelium (100\% cases without methylation). They also included the finding that the hypermethylated promoter of $R A R-\beta$ may block or interfere with the retinoid signaling pathways in NPC [30]. Later on, a strong association was shown between the expression of cyclooxygenase (COX-2) and loss of $R A R-\beta$ via the aberrant methylation in NPC tissues. Their data supported the finding that the inhibition of tumor development of $R A R-\beta$ may be related to its suppression of COX-2. The accumulation of COX-2 is related to tumor progression and invasiveness. Additionally, RAR- $\beta$ hypermethylation is correlated with histological type of NPC [36]. Challouf et al. (2012) also reported that aberrant methylation of $R A R-\beta$ was significantly frequent in NPC tumors with lymph node metastasis than those without metastasis [28]. Taken together, these data demonstrate that the loss expression of $R A R-\beta$ by the mechanism of hypermethylation is significantly associated with highly differentiated tumors, advanced tumor stage, and lymph node metastasis. The application of methylation markers of panel 
genes, including RASSF1A, RAR- $\beta, D A P K, p 16, p 15, p 14, M G M T$, and GSTP1, or RASSF1A, $R A R-\beta$, and $D A P K$, give a specificity of $100 \%$ and sensitivity of $100 \%[30,36]$. Thus, it indicates a promising methylation marker for NPC diagnosis.

LTF gene behaves as a tumor suppressor gene in NPC by the function of inducing cell rest and modulating the MAPK signaling pathway [14]. Other reports indicate that LTP also represses AKT signaling in NPC through multiple mechanisms [25]. Yi et al. (2006) first identified that the downregulation of LTF was observed in $76 \%$ (25 of 33 cases) of primary NPC tissues, and the hypermethylation of LTF was observed in $63.6 \%$ (21 of 33 cases) of primary NPC samples, but not in chronic nasopharyngeal tissues. By treatment of 5'-aza-2'deoxycytidine, the increasing transcript of LTF was found in the NPC cell line. Additionally, they also reported that two-hit silencing of LTF through genetic and epigenetic alterations may be a common and key role in nasopharyngeal tumorigenesis [23]. Thus, it may become a biologically relevant diagnosis marker for NPC.

\section{Conclusions and Future Perspectives}

The epigenetic event of hypermethylation plays key roles in nasopharyngeal tumorigenesis. Many studies have been carried out to identify the target TSGs located at $3 p$ which are responsible for NPC development and progress. In this study, we focused on exploiting the available data based on research over the past two decades to identify the potential TGS-located 3p-based methylation markers, particularly BLU, DLEC1, LTF, RASSF1A, and $R A R-\beta$, for NPC diagnosis. These profiles have become a potential component for the use of these TSGs in the design of in vitro assays and evaluation of further studies with a larger cohort of NPC patients to point out the perturbation targets in comparison with healthy volunteers as diagnostic markers in cancer management, including screening activities, monitoring of routine tumorigenesis, and development of TGS-located 3p-based therapies.

Author Contributions: All authors contributed to the design and conception of the study. writing original draft: T.D.L.; data collection and analysis: T.D.L. and T.N.N.; review and editing the manuscript: T.A.H.L. All authors have read and agreed to the published version of the manuscript.

Funding: This research was funded by Ho Chi Minh City Open University, Ho Chi Minh City, Vietnam under the grant number E2019.07.3.

Institutional Review Board Statement: Ethical review and approval were waived for this study.

Informed Consent Statement: Not applicable.

Data Availability Statement: Not applicable.

Acknowledgments: We express our special thanks to Ho Chi Minh City Open University for their genuine support throughout this research work under the grant number E2019.07.3.

Conflicts of Interest: The authors declare no conflict of interest.

\section{References}

1. Si, W.; Shen, J.; Zheng, H.; Fan, W. The role and mechanisms of action of microRNAs in cancer drug resistance. Clin. Epigenetics 2019, 11, 1-24. [CrossRef] [PubMed]

2. Tham, I.W.K.; Lu, J.J. Treatment of early stage nasopharyngeal carcinoma: Conventional versus new radiation therapy technologies. J. Radiat. Oncol. 2012, 1, 99-106. [CrossRef]

3. Tsao, S.W.; Yip, Y.L.; Tsang, C.M.; Pang, P.S.; Lau, V.M.Y.; Zhang, G.; Lo, K.W. Etiological factors of nasopharyngeal carcinoma. Oral Oncol. 2014, 50, 330-338. [CrossRef]

4. Sharma, S.; Kelly, T.K.; Jones, P.A. Epigenetics in cancer. Carcinogenesis 2009, 31, 27-36. [CrossRef]

5. Hutajulu, S.H.; Indrasari, S.R.; Indrawati, L.P.L.; Harijadi, A.; Duin, S.; Haryana, S.M.; Steenbergen, R.D.M.; Greijer, A.E.; Middeldorp, J.M. Epigenetic markers for early detection of nasopharyngeal carcinoma in a high risk population. Mol. Cancer 2011, 10, 48. [CrossRef]

6. Hong, L.L.; Lo, P.H.Y.; Xie, D.; Apte, S.S.; Cheung, A.K.L.; Cheng, Y.; Law, E.W.L.; Chua, D.; Zeng, Y.X.; Sai, W.T.; et al. Characterization of a novel epigenetically-silenced, growth-suppressive gene, ADAMTS9, and its association with lymph node metastases in nasopharyngeal carcinoma. Int. J. Cancer 2008, 123, 401-408. [CrossRef] 
7. Ayadi, W.; Karray-Hakim, H.; Khabir, A.; Feki, L.; Charfi, S.; Boudawara, T.; Ghorbel, A.; Daoud, J.; Frikha, M.; Busson, P.; et al. Aberrant methylation of p16, DLEC1, BLU and E-cadherin gene promoters in nasopharyngeal carcinoma biopsies from Tunisian patients. Anticancer Res. 2008, 28, 2161-2167. [PubMed]

8. Waddington, C.H. The Epigenotype. Int. J. Epidemiol. 2012, 41, 10-13. [CrossRef]

9. Han, B.; Yang, X.; Zhang, P.; Zhang, Y.; Tu, Y.; He, Z.; Li, Y.; Yuan, J.; Dong, Y.; Hosseini, D.K.; et al. DNA methylation biomarkers for nasopharyngeal carcinoma. PLoS ONE 2020, 15, 1-16. [CrossRef]

10. Thuy, L.H.A.; Thuan, L.D.; Phuong, T.K. DNA Hypermethylation in Breast Cancer. In Breast Cancer-From Biology to Medicine; InTech: London, UK, 2017.

11. Hui, A.B.-Y.; Lo, K.-W.; Leung, S.-F.; Teo, P.; Fung, M.K.F.; To, K.F.; Wong, N.; Choi, P.H.K.; Lee, J.C.K.; Huang, D.P. Detection of recurrent chromosomal gains and losses in primary nasopharyngeal carcinoma by comparative genomic hybridisation. Int. J. Cancer 1999, 82, 498-503. [CrossRef]

12. Li, X.; Wang, E.; Zhao, Y.; Ren, J.-Q.; Jin, P.; Yao, K.-T.; Marincola, F.M. Chromosomal imbalances in nasopharyngeal carcinoma: A meta-analysis of comparative genomic hybridization results. J. Transl. Med. 2006, 4, 4. [CrossRef] [PubMed]

13. Chow, L.S.N.; Lo, K.W.; Kwong, J.; To, K.F.; Tsang, K.S.; Lam, C.W.; Dammann, R.; Huang, D.P. RASSF1A is a target tumor suppressor from 3p21.3 in nasopharyngeal carcinoma. Int. J. Cancer 2004, 109, 839-847. [CrossRef]

14. Chen, J.; Fu, L.; Zhang, L.Y.; Kwong, D.L.; Yan, L.; Guan, X.Y. Tumor suppressor genes on frequently deleted chromosome 3p in nasopharyngeal carcinoma. Chin. J. Cancer 2012, 31, 215-222. [CrossRef]

15. Nawaz, I.; Hu, L.F.; Du, Z.M.; Moumad, K.; Ignatyev, I.; Pavlova, T.V.; Kashuba, V.; Almgren, M.; Zabarovsky, E.R.; Ernberg, I. Integrin $\alpha 9$ gene promoter is hypermethylated and downregulated in nasopharyngeal carcinoma. Oncotarget 2015, 6, 31493-31507. [CrossRef] [PubMed]

16. Wing, L.Y.; Hong, L.L.; Zabarovsky, E.R.; Lerman, M.I.; Sham, J.S.T.; Chua, D.T.T.; Sai, W.T.; Stanbridge, E.J.; Lung, M.L. Functional studies of the chromosome 3p21.3 candidate tumor suppressor gene BLU/ZMYND10 in nasopharyngeal carcinoma. Int. J. Cancer 2006, 119, 2821-2826. [CrossRef]

17. Qiu, G.H.; Tan, L.K.S.; Loh, K.S.; Lim, C.Y.; Srivastava, G.; Tsai, S.T.; Tsao, S.W.; Tao, Q. The candidate tumor suppressor gene BLU, located at the commonly deleted region 3p21.3, is an E2F-regulated, stress-responsive gene and inactivated by both epigenetic and genetic mechanisms in nasopharyngeal carcinoma. Oncogene 2004, 23, 4793-4806. [CrossRef] [PubMed]

18. Leung Cheung, A.K.; Lung, H.L.; Hung, S.C.; Law, E.W.L.; Cheng, Y.; Yau, W.L.; Bangarusamy, D.K.; Miller, L.D.; Liu, E.T.B.; Shao, J.Y.; et al. Functional analysis of a cell cycle-associated, tumor-suppressive gene, protein tyrosine phosphatase receptor type G, in nasopharyngeal carcinoma. Cancer Res. 2008, 68, 8137-8145. [CrossRef] [PubMed]

19. Liu, X.Q.; Chen, H.K.; Zhang, X.S.; Pan, Z.G.; Li, A.; Feng, Q.S.; Long, Q.X.; Wang, X.Z.; Zeng, Y.X. Alterations of BLU, a candidate tumor suppressor gene on chromosome 3p21.3, in human nasopharyngeal carcinoma. Int. J. Cancer 2003, 106, 60-65. [CrossRef]

20. Lao, T.D.; Huyen Le, T.A. Characteristic of ZMYND10 Gene's Promoter Hypermethylation in Nasopharyngeal Carcinoma Biopsies from Vietnamese Patients. Asian J. Pharm. Res. Health Care 2019, 10, 60-65. [CrossRef]

21. Yi, H.-M.; Ren, C.-P.; Peng, D.; Zhou, L.; Li, H.; Yao, K.-T. [Expression, loss of heterozygosity, and methylation of GNAT1 gene in nasopharyngeal carcinoma]. Ai Zheng 2007, 26, 9-14.

22. Zhou, W.; Feng, X.; Li, H.; Wang, L.; Zhu, B.; Liu, W.; Zhao, M.; Yao, K.; Ren, C. Inactivation of LARS2, located at the commonly deleted region 3p21.3, by both epigenetic and genetic mechanisms in nasopharyngeal carcinoma. Acta Biochim. Biophys. Sin. 2009, 41, 54-62. [CrossRef]

23. Yi, H.-M.; Li, H.; Peng, D.; Zhang, H.-J.; Wang, L.; Zhao, M.; Yao, K.-T.; Ren, C.-P. Genetic and epigenetic alterations of LTF at 3p21.3 in nasopharyngeal carcinoma. Oncol. Res. 2006, 16, 261-272. [CrossRef]

24. Zhang, H.; Feng, X.; Liu, W.; Jiang, X.; Shan, W.; Huang, C.; Yi, H.; Zhu, B.; Zhou, W.; Wang, L.; et al. Underlying mechanisms for LTF inactivation and its functional analysis in nasopharyngeal carcinoma cell lines. J. Cell. Biochem. 2011, 112, 1832-1843. [CrossRef]

25. Deng, M.; Zhang, W.; Tang, H.; Ye, Q.; Liao, Q.; Zhou, Y.; Wu, M.; Xiong, W.; Zheng, Y.; Guo, X.; et al. Lactotransferrin acts as a tumor suppressor in nasopharyngeal carcinoma by repressing AKT through multiple mechanisms. Oncogene 2013, 32, 4273-4283. [CrossRef] [PubMed]

26. Wong, T.S.; Kwong, D.L.W.; Sham, J.S.T.; Wei, W.I.; Kwong, Y.L.; Yuen, A.P.W. Quantitative Plasma Hypermethylated DNA Markers of Undifferentiated Nasopharyngeal Carcinoma. Clin. Cancer Res. 2004, 10, 2401-2406. [CrossRef] [PubMed]

27. Wong, T.S.; Tang, K.C.; Kwong, D.L.W.; Sham, J.S.T.; Wei, W.I.; Kwong, Y.L.; Yuen, A.P.W. Differential gene methylation in undifferentiated nasopharyngeal carcinoma. Int. J. Oncol. 2003, 22, 869-874. [CrossRef]

28. Challouf, S.; Ziadi, S.; Zaghdoudi, R.; Ksiaa, F.; Ben Gacem, R.; Trimeche, M. Patterns of aberrant DNA hypermethylation in nasopharyngeal carcinoma in Tunisian patients. Clin. Chim. Acta 2012, 413, 795-802. [CrossRef]

29. Chang, H.W.; Chan, A.; Kwong, D.L.W.; Wei, W.I.; Suam, J.S.T.; Yuen, A.P.W. Evaluation of hypermethylated tumor suppressor genes as tumor markers in mouth and throat rinsing fluid, nasopharyngeal swab and peripheral blood of nasopharygeal carcinoma patient. Int. J. Cancer 2003, 105, 851-855. [CrossRef]

30. Kwong, J.; Lo, K.W.; To, K.F.; Teo, P.M.L.; Johnson, P.J.; Poon Huang, D. Promoter hypermethylation of multiple genes in nasopharyngeal carcinoma. Clin. Cancer Res. 2002, 8, 131-137. 
31. Tong, J.H.M.; Tsang, R.K.Y.; Lo, K.-W.; Woo, J.K.S.; Kwong, J.; Chan, M.W.Y.; Chang, A.R.; van Hasselt, C.A.; Huang, D.P.; To, K.-F. Quantitative Epstein-Barr virus DNA analysis and detection of gene promoter hypermethylation in nasopharyngeal (NP) brushing samples from patients with NP carcinoma. Clin. Cancer Res. 2002, 8, 2612-2619. [PubMed]

32. Zhang, Z.; Sun, D.; Hutajulu, S.H.; Nawaz, I.; Nguyen Van, D.; Huang, G.; Haryana, S.M.; Middeldorp, J.M.; Ernberg, I.; Hu, L.F. Development of a Non-Invasive Method, Multiplex Methylation Specific PCR (MMSP), for Early Diagnosis of Nasopharyngeal Carcinoma. PLoS ONE 2012, 7, 1-6. [CrossRef]

33. Wang, T.; Liu, H.; Chen, Y.; Liu, W.; Yu, J.; Wu, G. Methylation associated inactivation of RASSF1A and its synergistic effect with activated K-Ras in nasopharyngeal carcinoma. J. Exp. Clin. Cancer Res. 2009, 28. [CrossRef]

34. Lo, K.W.; Kwong, J.; Hui, A.B.; Chan, S.Y.; To, K.F.; Chan, A.S.; Chow, L.S.; Teo, P.M.; Johnson, P.J.; Huang, D.P. High frequency of promoter hypermethylation of RASSF1A in nasopharyngeal carcinoma. Cancer Res. 2001, 61, 3877-3881.

35. Thieu, H.H.; Lao, T.D.; Le, T.A.H. Characterization of Promoter Hypermethylation of Tumor Suppressor Gene Rassf1a and Its Association With the Risk of Nasopharyngeal Carcinoma. Pharmacophore 2020, 11, 56-62.

36. Fendri, A.; Masmoudi, A.; Khabir, A.; Sellami-Boudawara, T.; Daoud, J.; Frikha, M.; Ghorbel, A.; Gargouri, A.; Mokdad-Gargouri, R. Inactivation of RASSF1A, RAR $\beta 2$ and DAP-kinase by promoter methylation correlates with lymph node metastasis in nasopharyngeal carcinoma. Cancer Biol. Ther. 2009, 8, 444-451. [CrossRef] [PubMed]

37. Zhou, L.; Jiang, W.; Ren, C.; Yin, Z.; Feng, X.; Liu, W.; Taoz, Q.; Yao, K. Frequent hypermethylation of RASSF1A and TSLC1, and high, viral load of Epstein-Barr virus DNA in nasopharyngeal carcinoma and matched tumor-adjacent tissues. Neoplasia 2005, 7, 809-815. [CrossRef] [PubMed]

38. Tian, F.; Yip, S.P.; Kwong, D.L.W.; Lin, Z.; Yang, Z.; Wu, V.W.C. Promoter hypermethylation of tumor suppressor genes in serum as potential biomarker for the diagnosis of nasopharyngeal carcinoma. Cancer Epidemiol. 2013, 37, 708-713. [CrossRef]

39. Loyo, M.; Brait, M.; Kim, M.S.; Ostrow, K.L.; Jie, C.C.; Chuang, A.Y.; Califano, J.A.; Liégeois, N.J.; Begum, S.; Westra, W.H.; et al. A survey of methylated candidate tumor suppressor genes in nasopharyngeal carcinoma. Int. J. Cancer 2011, 128, 1393-1403. [CrossRef]

40. Kwong, J.; Chow, L.S.-N.; Wong, A.Y.-H.; Hung, W.-K.; Chung, G.T.-Y.; To, K.-F.; Chan, F.L.; Daigo, Y.; Nakamura, Y.; Huang, D.P.; et al. Epigenetic inactivation of the deleted in lung and esophageal cancer 1 gene in nasopharyngeal carcinoma. Genes Chromosom. Cancer 2007, 46, 171-180. [CrossRef]

41. Kwong, J.; Lo, K.-W.; Chow, L.S.-N.; Chan, F.L.; To, K.-F.; Huang, D.P. Silencing of the retinoid response gene TIG1 by promoter hypermethylation in nasopharyngeal carcinoma. Int. J. Cancer 2005, 113, 386-392. [CrossRef] [PubMed]

42. Dammann, R.; Li, C.; Yoon, J.-H.; Chin, P.L.; Bates, S.; Pfeifer, G.P. Epigenetic inactivation of a RAS association domain family protein from the lung tumour suppressor locus 3p21.3. Nat. Genet. 2000, 25, 315-319. [CrossRef] [PubMed]

43. Lo, K.W.; To, K.F.; Huang, D.P. Focus on nasopharyngeal carcinoma. Cancer Cell 2004, 5, 423-428. [CrossRef]

44. Shivakumar, L.; Minna, J.; Sakamaki, T.; Pestell, R.; White, M.A. The RASSF1A tumor suppressor blocks cell cycle progression and inhibits cyclin D1 accumulation. Mol. Cell. Biol. 2002, 22, 4309-4318. [CrossRef] [PubMed]

45. Ye, M.; Huang, T.; Ni, C.; Yang, P.; Chen, S. Diagnostic Capacity of RASSF1A Promoter Methylation as a Biomarker in Tissue, Brushing, and Blood Samples of Nasopharyngeal Carcinoma. EBioMedicine 2017, 18, 32-40. [CrossRef] [PubMed]

46. Ooft, M.L.; van Ipenburg, J.; van Loo, R.; de Jong, R.; Moelans, C.; Braunius, W.; de Bree, R.; van Diest, P.; Koljenović, S.; Baatenburg de Jong, R.; et al. Molecular profile of nasopharyngeal carcinoma: Analysing tumour suppressor gene promoter hypermethylation by multiplex ligation-dependent probe amplification. J. Clin. Pathol. 2018, 71, 351-359. [CrossRef]

47. Zhang, S.-Q.; Pan, S.-M.; Liang, S.-X.; Han, Y.-S.; Chen, H.-B.; Li, J.-C. Research status and prospects of biomarkers for nasopharyngeal carcinoma in the era of high-throughput omics (Review). Int. J. Oncol. 2021, 58, 9. [CrossRef]

48. Gihbid, A.; Benzeid, R.; Faouzi, A.; Nourlil, J.; Tawfiq, N.; Benchakroun, N.; Guensi, A.; Bendahhou, K.; Benider, A.; El Benna, N.; et al. Circulating cell-free epstein-barr virus DNA levels and clinical features in Moroccan patients with nasopharyngeal carcinoma. Infect. Agent. Cancer 2021, 16, 15. [CrossRef]

49. Skalska, L.; White, R.E.; Franz, M.; Ruhmann, M.; Allday, M.J. Epigenetic Repression of p16INK4A by Latent Epstein-Barr Virus Requires the Interaction of EBNA3A and EBNA3C with CtBP. PLoS Pathog. 2010, 6, e1000951. [CrossRef]

50. Tsai, C.-L.; Li, H.-P.; Lu, Y.-J.; Hsueh, C.; Liang, Y.; Chen, C.-L.; Tsao, S.W.; Tse, K.-P.; Yu, J.-S.; Chang, Y.-S. Activation of DNA Methyltransferase 1 by EBV LMP1 Involves c-Jun NH 2 -Terminal Kinase Signaling. Cancer Res. 2006, 66, 11668-11676. [CrossRef]

51. Dai, W.; Zheng, H.; Cheung, A.K.L.; Lung, M.L. Genetic and epigenetic landscape of nasopharyngeal carcinoma. Chinese Clin. Oncol. 2016, 5, 16. [CrossRef]

52. García-Gutiérrez, L.; McKenna, S.; Kolch, W.; Matallanas, D. RASSF1A Tumour Suppressor: Target the Network for Effective Cancer Therapy. Cancers 2020, 12, 229. [CrossRef]

53. Stresemann, C.; Lyko, F. Modes of action of the DNA methyltransferase inhibitors azacytidine and decitabine. Int. J. Cancer 2008, 123, 8-13. [CrossRef] [PubMed]

54. Jiang, W.; Li, Y.-Q.; Liu, N.; Sun, Y.; He, Q.-M.; Jiang, N.; Xu, Y.-F.; Chen, L.; Ma, J. 5-Azacytidine Enhances the Radiosensitivity of CNE2 and SUNE1 Cells In Vitro and In Vivo Possibly by Altering DNA Methylation. PLoS ONE 2014, 9, e93273. [CrossRef] [PubMed]

55. Liang, Y.Y.; Deng, X.B.; Lin, X.T.; Jiang, L.L.; Huang, X.T.; Mo, Z.W.; Yuan, Y.W.; Teh, M.T. RASSF1A inhibits PDGFB-driven malignant phenotypes of nasopharyngeal carcinoma cells in a YAP1-dependent manner. Cell Death Dis. 2020, 11, 1-12. [CrossRef] [PubMed] 